Faculty of Science

Faculty Publications

This is a post-review version of the following article:

The role of available sites in the activity of lattice gases with geometric constraints Jonathan R. Strobl and David A. Harrington

2013

The final published version of this article can be found at:

https://doi.org/10.1063/1.4820400

Citation for this paper:

Strobl, J.R. \& Harrington, D.A. (2013). The role of available sites in the activity of lattice gases with geometric constraints. The Journal of Chemical Physics, 139, 104104. https://doi.org/10.1063/1.4820400 


\title{
The role of available sites in the activity of lattice gases with geometric constraints
}

\author{
Jonathan R. Strobl ${ }^{1}$ and David A. Harrington ${ }^{1, \text { a) }}$
}

Chemistry Department, University of Victoria, Victoria, BC, V8W 3P6, Canada

(Date textdate; Received textdate; Revised textdate; Accepted textdate;

Published textdate)

The activity in lattice-gas systems with geometric constraints is shown to be the ratio of the number of particles to the number of available sites. The key role of sites available for occupation is emphasized. Available sites may be different for different species and are not necessarily just unoccupied sites. Location-specific or non-local constraints are allowed. An analytical expression for the number of available sites is given for the hard-hexagon model. The utility of an expression for available sites is illustrated for the non-trivial case of a mixed Langmuir/hard-hexagon adsorption system, where the influence of the Langmuir adsorbates on the hard-hexagon phase transition is investigated. The dependence on available sites indicates how to extend these results to the kinetic regime, and simulations of kinetic voltammograms for the hard-hexagon model are given as an example.

a) Electronic mail: dharr@uvic.ca 
Role of available sites

\section{INTRODUCTION}

This paper concerns lattice-gas systems with only geometric constraints, i.e., systems where the configurations with given numbers of particles of various species all have the same energy. The geometric constraints may be non-trivial, for example, type A particles may be forbidden from occupying sites adjacent to type A particles, but may be allowed to be adjacent to type $\mathrm{B}$ particles; these types of interactions are the lattice equivalent of non-additive exclusion interactions in continuous hard-sphere models. Locality-specific constraints are also allowed, e.g., a line of lattice sites where occupancy is forbidden for one or more species. A simple statistical-mechanical derivation is first used to show that the activity is the ratio of the number of particles to the number of available sites. The terminology of 2-D adsorption systems will be used here: counts of adsorbed species or available sites are normalized by the number of lattice sites and written as coverages $\theta$ or $\theta_{\mathrm{av}}$, and so we write the activity $a=\theta / \theta_{\mathrm{av}}$. However, the arguments may be applied also to 3-D systems and systems that do not have the adsorption sites strictly arrayed in a lattice.

The key role of $\theta_{\mathrm{av}}$, the sites available for occupation, is emphasized. (Throughout this paper, adsorption occurs at a specific site or location, which counts as one even though it may cover or exclude more than one lattice site.) Available sites may be different for different species and are not necessarily just unoccupied lattice sites. The key role of available sites is the lattice-gas equivalent of the key role of spare or available volume in the statistical geometry of continuous hard-sphere systems ${ }^{1-4}$. Our derivation of the activity is an extension to lattice gases of the derivation of Speedy for hard spheres ${ }^{1}$ or of Corti and Bowles for hardsphere mixtures ${ }^{4}$. Derivations directly linking addition of a particle to chemical potential, as here, originate with Widom's particle insertion method ${ }^{5}$ Widom's method was applied to lattice gas systems before ${ }^{6-9}$, but the key significance of the number of available sites did not seem to be recognized. The work by Soto-Campos et. al. ${ }^{10}$ does not use the particle addition method, but does point out the key role of the available sites for hard rod systems. They conjectured that their equivalent of $a=\theta / \theta_{\mathrm{av}}$ had more universal validity, but proved it only for hard-rods on a 1-D lattice.

A simple derivation of the Langmuir adsorption isotherm is a trivial result of the theory, but the theory applied to the hard hexagon model and mixed hard hexagon/Langmuir systems provides non-trivial applications. The hard hexagon model describes adsorption on 


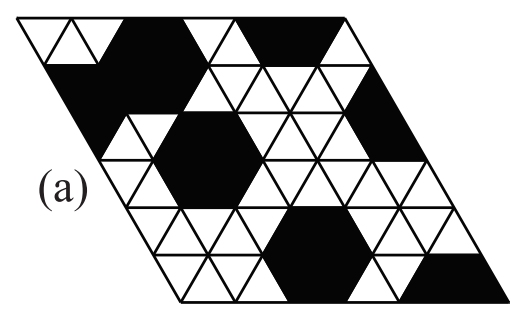

(b)

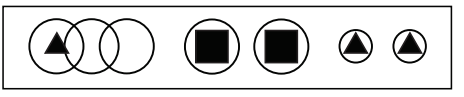

FIG. 1. Surface configurations. (a) A configuration of hard hexagons on a triangular lattice of $M=36$ sites with $N=5$ adsorbed species (black hexagons), $N_{\mathrm{av}}=6$ available sites (vertices not touching hexagons) and 25 forbidden lattice sites (at the vertices of the hexagons). Periodic boundary conditions mean the right and bottom edges are duplicates of the left and top edges. (b) A configuration of an artificial surface discussed in the text, with $M=7, N_{\boldsymbol{\Xi}}=2, N_{\mathbf{\Lambda}}=3$, $N_{\mathrm{av}, \boldsymbol{\square}}=1$ (site 3 only), $N_{\mathrm{av}, \boldsymbol{\Lambda}}=2$ (sites 2 and 3$)$.

a triangular lattice in which nearest neighbor adsorption is forbidden, so that the "exclusion zone" around an adsorbate is a hexagon. This is one of the few statistical-mechanical lattice models with an exact solution, found by Baxter ${ }^{11,12}$, and recast in an algebraic form by Joyce $^{13,14}$. There is a phase transition from 2-D gas to coexistence of 2-D solid and gas phases when the coverage exceeds a critical coverage $\theta_{c}=0.276$. The complexity of the model arises from the fact that different configurations with the same number of adsorbates nonetheless have different numbers of available sites for further adsorption, because the number of unoccupied lattice sites adjacent to adsorbates (forbidden lattice sites) depends on the configuration (Fig. 1(a)).

The hard hexagon model is of interest for two reasons. Firstly, it appears to explain some experimental results in surface science and electrochemistry ${ }^{15-20}$. Secondly, as a model system with a phase transition and an analytical solution, it can be used to verify methodologies independently of any experimental or theoretical approximations. For example, Nagai ${ }^{21}$ used it as a test case to decide between two versions of transition-state theory in thermal desorption spectroscopy. In electrochemistry, the mean-field solution of the Ising lattice-gas model with nearest-neighbour interactions of energy $\varepsilon_{n n}$ is known as the Frumkin isotherm 
and is frequently used to simulate voltammograms with phase transitions. However, the well-known deficiencies of the mean-field model mean that the mean-field phase transition at the non-dimensionalized interaction (Frumkin) parameter $g=c \varepsilon_{n n} / k T=-4$ is far from the true (square lattice, coordination number $c=4$ ) value of $g=-7.05^{22}$. Therefore inadequacies of fits of the Frumkin model to experimental data may be due either in deficiencies in the mean-field approximation, or that the underlying nearest-neighbor interaction model does not apply in the experimental system. Nonetheless, the Frumkin model remains widely used simply because it has an analytical solution, and alternatives such as Monte-Carlo calculations are more complex and slower computationally. This is especially an issue in voltammetry or thermal desorption spectroscopy, as these methods require solutions over the whole coverage range.

Armed with an analytical expression for $\theta_{\mathrm{av}}$ for the hard hexagon model, we show how to combine hard-hexagon adsorbates with Langmuir adsorbates in a competitive adsorption model. An interesting aspect of this is how the Langmuir adsorbates may disrupt the phase transition. There are only a few rigorous results for phase transitions of lattice gas mixtures,

e.g., ${ }^{23-25}$ We also formulate a kinetic version of the hard-hexagon model that requires only solution of an ordinary differential equation to determine coverage-time behavior, and use it to simulate voltammograms for electrochemical adsorption.

\section{THEORY}

We consider a lattice gas model with the following assumptions:

1. The temperature and area of the surface (number of surface sites on the empty surface, $M)$ are fixed. The Helmholtz energy $A$ is therefore the appropriate free energy variable.

2. The internal motions of the adsorbates are independent of any interaction energies between them.

3. The chemical potential is defined as the work to add a particle (adsorbate) of a given type $i$ to the system, $\mu_{i}=A_{N_{i}+1}-A_{N_{i}}$, even for a system with finite numbers of sites and adsorbates.

4. Geometric constraints forbidding adsorption at certain locations are allowed, but addition of a particle of type $i$ to any of the locations available to it leads to the same 
Role of available sites

change in energy.

Here we consider two canonical ensembles with fixed particle numbers for all $s$ species, one with particle counts $N_{1}, N_{2}, \ldots, N_{s}$ and partition function $Q\left(N_{1}, N_{2}, \ldots, N_{s}\right)$ that we abbreviate $Q_{N_{i}}$, and another with one particle of species $i$ added, $Q\left(N_{1}, \ldots, N_{i-1}, N_{i}+\right.$ $\left.1, N_{i+1}, \ldots, N_{s}\right)$ that we abbreviate $Q_{N_{i}+1}$. Similar abbreviated notation is used for the number of configurations, $\Omega_{N_{i}}$ or $\Omega_{N_{i}+1}$ and Helmholtz energies, $A_{N_{i}}$ or $A_{N_{i}+1}$.

Assumptions 1 and 2 are conventional for lattice gas theory. The philosophy here is slightly different from Widom's, in that we take the chemical potential expression $\mu_{i}=$ $A_{N_{i}+1}-A_{N_{i}}$ (assumption 3) to be exact for a finite system, as is occasionally done, e.g. ${ }^{26-29}$. In the thermodynamic limit of large $N_{i}$ this definition agrees with the usual one, because it is just the finite difference approximation to the derivative.

$$
\begin{gathered}
\mu_{i}=A_{N_{i}+1}-A_{N_{i}}=\frac{A_{N_{i}+1}-A_{N_{i}}}{\left(N_{i}+1\right)-N_{i}} \\
\underset{N_{i}, N_{j \neq i}, M=\infty}{\longrightarrow}\left(\frac{\partial A}{\partial N_{i}}\right)_{T, M, N_{j \neq i}}
\end{gathered}
$$

The limit is taken for $N_{i} \rightarrow \infty, N_{j} \rightarrow \infty, M \rightarrow \infty$ with $\theta_{i}=N_{i} / M$ and the other particle coverages $\theta_{j}=N_{j} / M$ constant. Han et $\mathrm{al}^{30}$, in a more typical approach, have discussed different finite difference approximations to the "real" (partial-derivative) chemical potential. Since we are developing analytical results only in the thermodynamic limit, the philosophical issue is immaterial for practical purposes. However, we suggest that deriving the chemical potential exactly for a finite system, and then approaching the thermodynamic limit, instead of finding the free energy in the thermodynanic limit and then differentiating to find the chemical potential, may offer insights in solving exactly some statistical models of interest. The geometric constraints referred to in assumption 4 are effectively infinite energy repulsions that do not introduce an energy parameter into the problem. These are similar to the hard-wall repulsions or volume exclusion interactions in continuous hardparticle systems. The possibilities and limitations of these constraints will be discussed in more detail in Sec. III A.

According to assumptions 2 and 4, the canonical partition function is given by Eq. (3), where $\Omega_{N_{i}}$ is the number of ways of arranging all adsorbates on the surface, i.e., $k \ln \Omega_{N_{i}}$ is the configurational entropy for the system. (The adsorption energies $\varepsilon_{i}, \varepsilon_{j}$ could alternatively be included in the molecular partition functions $\left.q_{i}, q_{j}\right)$. Proceding to calculate the chemical 
potential from the Helmholtz energy, Eq. (4), using $\mu_{i}=A_{N_{i}+1}-A_{N_{i}}$ and keeping $T, M, N_{j}$ constant gives Eq. (5), and the activity takes the particularly simple form of Eq. (6)

$$
\begin{aligned}
Q_{N_{i}} & =\Omega_{N_{i}}\left(q_{i} \exp \left(-\varepsilon_{i} / k T\right)\right)^{N_{i}} \prod_{j \neq i}\left(q_{j} \exp \left(-\varepsilon_{j} / k T\right)\right)^{N_{j}} \\
A_{N_{i}} & =-k T \ln Q_{N_{i}} \\
\mu_{i} & =A_{N_{i}+1}-A_{N_{i}}=\left(-k T \ln q_{i}+\varepsilon_{i}\right)+k T \ln \left(\Omega_{N_{i}} / \Omega_{N_{i}+1}\right) \\
& =\mu_{i}^{\mathrm{o}}+k T \ln \left(\Omega_{N_{i}} / \Omega_{N_{i}+1}\right) \\
a_{i, N_{i}} & =\Omega_{N_{i}} / \Omega_{N_{i}+1}
\end{aligned}
$$

As an immediate application of this, consider the case of addition of one Langmuir adsorbate (no lateral interactions) to a system of $N$ such adsorbates (the subscript $i$ is redundant here since there is only one species). Then $\Omega_{N}=\left(\begin{array}{l}M \\ N\end{array}\right)$ and the activity is given by

$$
a_{N}=\left(\begin{array}{c}
M \\
N
\end{array}\right) /\left(\begin{array}{c}
M \\
N+1
\end{array}\right)=\frac{N+1}{M-N}=\frac{(N+1) / M}{1-N / M} .
$$

The numerator is the coverage for the system with $N+1$ adsorbates, i.e., after addition, and we use a prime to distinguish this from the coverage before addition, $\theta$. The activity for the finite system is therefore

$$
a_{N}=\frac{\theta^{\prime}}{1-\theta}
$$

Given assumption 3, this is an exact result. In the thermodynamic limit, there is no distinction between $\theta^{\prime}$ and $\theta$, and we arrive at the well-known result $a=\theta /(1-\theta)$, derived rather simply and without recourse to Stirling's approximation. A similar calculation for two types of Langmuir adsorbates A and B competing for the same sites leads to $a_{\mathrm{A}, N}=\theta_{\mathrm{A}}^{\prime} /\left(1-\theta_{\mathrm{A}}-\theta_{\mathrm{B}}\right)$ and $a_{\mathrm{B}, N}=\theta_{\mathrm{B}}^{\prime} /\left(1-\theta_{\mathrm{A}}-\theta_{\mathrm{B}}\right)$.

We now show that rather generally the activity is given by $\theta_{i}^{\prime} /\left\langle\theta_{\mathrm{av}, i}\right\rangle$, where the angle brackets denote an ensemble average for the system with $N_{i}$ adsorbates. Consider a configuration $k$ of the system containing $N_{i}$ adsorbates that has $N_{\mathrm{av}, i}(k)$ possible locations to add the next adsorbate. Adsorbate here refers to the type for which we are to calculate the activity; there may be many other types of adsorbates, provided the number of each does not vary in the different configurations. Add up all the available sites from all the $\Omega_{N_{i}}$ configurations, to give an ensemble total $T_{\mathrm{av}, i}=\sum_{k=1}^{\Omega_{N_{i}}} N_{\mathrm{av}, i}(k)$. Now make new configurations by adding an adsorbate to each of these $T_{\mathrm{av}, i}$ sites. Each of the resulting $T_{\mathrm{av}, i}$ configurations will 
be identical to $N_{i}$ others, because any of the indistinguishable $N_{i}+1$ adsorbates could have been the one just added. So the correct number of configurations with $N_{i}+1$ adsorbates is

$$
\Omega_{N_{i}+1}=T_{\mathrm{av}, i} /\left(N_{i}+1\right) .
$$

The ensemble average of the number of available sites before the addition is $\left\langle N_{\mathrm{av}, i}\right\rangle=$ $T_{\mathrm{av}, i} / \Omega_{N_{i}}$ and this may be combined with the above equation to give

$$
\frac{\Omega_{N_{i}+1}}{\Omega_{N_{i}}}=\frac{\left\langle N_{\mathrm{av}, i}\right\rangle}{N_{i}+1} .
$$

Combining with Eq. (6) leads to a simple expression for the activity of species $i$ for the finite system that has $N_{i}$ particles of species $i$

$$
\begin{aligned}
a_{i, N_{i}} & =\frac{\Omega_{N_{i}}}{\Omega_{N_{i}+1}}=\frac{N_{i}+1}{\left\langle N_{\mathrm{av}, i}\right\rangle}=\frac{\left(N_{i}+1\right) / M}{\left\langle N_{\mathrm{av}, i}\right\rangle / M} \\
& =\frac{\theta_{i}^{\prime}}{\left\langle\theta_{\mathrm{av}, i}\right\rangle} .
\end{aligned}
$$

Note that the use of the average here does not involve any approximation. In the thermodynamic limit, this reduces to

$$
a_{i}=\frac{\theta_{i}}{\theta_{\mathrm{av}, i}}
$$

where $\theta_{\mathrm{av}, i}$ means coverage of sites available for adsorption of species $i$.

\section{RESULTS AND DISCUSSION}

\section{A. The nature of available sites and geometric constraints}

We begin with a discussion of the limitations and possibilities of assumption 4 . The simplest type of geometric constraint is that a lattice site already occupied is not available for further adsorption, which of course is the basis for the Langmuir adsorption model. More interesting are cases where adsorption on a lattice site excludes further adsorption on any other specified sites, such as the nearest-neighbor exclusions in the hard hexagon model. Richer possibilities are also entertained, as best demonstrated in terms of a small artificial example with non-trivial possibilities. (Further details of this example, including a figure showing all configurations, are given in the supplementary material ${ }^{31}$.) Fig. 1(b) shows one configuration for a surface with seven sites (circles), two types of adsorbates (squares or triangles), and the following rules: Squares have hard repulsions between each other and 
the triangles to the extent that for the leftmost three sites (overlapping circles), they cannot occupy neighboring sites. (Two triangles may be adjacent on these sites, though.) For the next two sites, either squares or triangles may adsorb without any restrictions. For the last two sites, only triangles may adsorb, again without restrictions. The configuration shown is one of 32 configurations with two squares and three triangles obeying these rules. If we now consider addition of a square to this system, there are 12 available sites amongst the 32 configurations that are suitable, so according to Eq. (9), there will be $12 / 3=4$ configurations with 3 squares and 3 triangles, as is easily verified. Similarly, for addition of a triangle to this system, there are 40 available sites amongst the 32 configurations that are suitable, leading to $40 / 4=10$ configurations with 2 squares and 4 triangles.

This example emphasizes that the meaning of "available" in the above derivation means available for adsorption of the type of adsorbate for which we are calculating the activity. Available is not the same as "free" in the sense of an unoccupied lattice site, and the available sites may be different for different adsorbates. (Fig. 1(b) has two unoccupied sites; both are available for adsorption of a triangle, but only one is available for adsorption of a square.) Different configurations may have different numbers of available sites, and there may be more that one type of site for adsorption, e.g., the two types of circles for triangle adsorption. This flexibility in site type is constrained by the requirement that there be the same adsorption energy for the different sites on which a species may adsorb. A practical example where rules like this might apply is adsorption on sites near a step edge, where large molecules might be excluded but smaller molecules might not be. Just as constraints may be location specific, they may also be non-local, e.g., specifying that particles may not be five lattice sites apart. There is no requirement that sites have any particular spatial relationship to each other, and so the derivation does not actually require a lattice gas system. The sites

must be distinguishable: two sites at the same location are not allowed, or equivalently, adsorption of two particles of the same type at the same site is not allowed.

\section{B. Hard hexagons}

The above derivation shows that the activity depends only on the average number of available sites, and not their distribution across members of the ensemble. The analytical solution of the hard-hexagon model expresses the activity, free energy per lattice site, or 


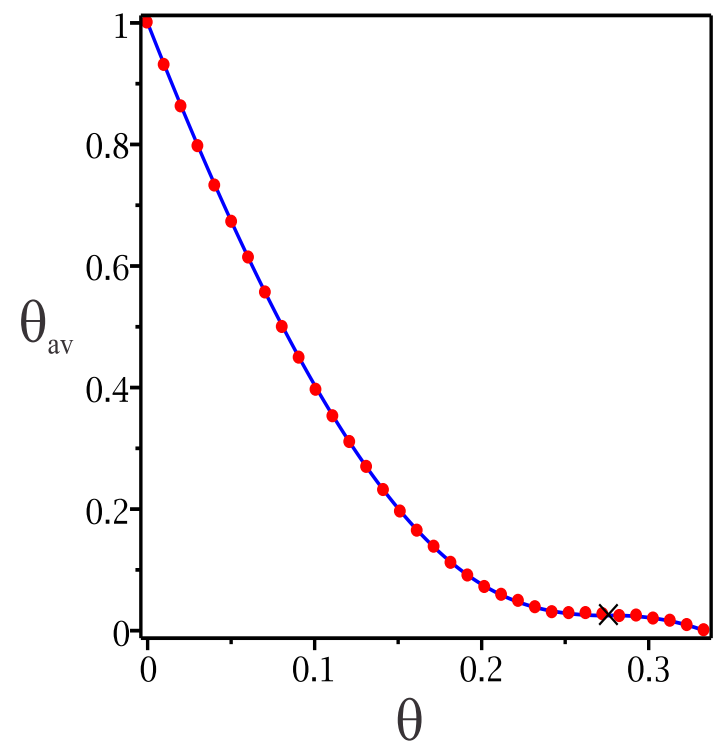

FIG. 2. Available sites for the hard hexagon model. Solid line: analytical solution; the cross shows the phase transition point. Circles: result of Monte-Carlo simulations (every 11th point shown).

compressibility in terms of the coverage, and makes no reference to the available or forbidden sites. However, since the activity of the hexagons as a function of coverage, $a(\theta)$, is available, Eq. (12) immediately gives an exact analytical expression for the coverage of available sites for hard hexagons, $\theta_{\mathrm{av}}=\theta / a(\theta)$. The coverage $\theta_{\mathrm{av}}$ is given explicitly as a function of $\theta$ in the supplementary material ${ }^{31}$, and is plotted in Fig. 2 . Taking the derivative at $\theta=0$ yields the expected value of -7 : the addition of an isolated adsorbate (hexagon) to an empty lattice removes the central adsorption site and the six nearest neighbors. Similarly, the derivative at $\theta=1 / 3$ is -1 , consistent with filling the last, single-lattice-site holes in the solid-like adlayer.

To further verify that this expression for $\theta_{\mathrm{av}}$ represents the sites available for adsorption, Monte Carlo simulations were carried out on a $33 \times 33$ triangular lattice with periodic boundary conditions. Constant-coverage simulations were carried out for all numbers of adsorbates from two to maximum coverage. The final state of the surface after each simulation was used as the initial state for the sucessive one. The simulations consisted of first adsorbing the new adsorbate, followed by $1000 \sqrt{N}$ migration steps in which a randomly selected adsorbate could move to a randomly-chosen available neighboring lattice site. The average coverage of available sites agrees with the analytical function to within \pm 0.008 , Fig. 2 . 
The Monte-Carlo simulations validate the meaning of $\theta_{\mathrm{av}}$ in the analytical expresssion, but involve some level of approximation. To verify that Eq. (9) is exact, it was tested by generating all configurations for a $6 \times 6$ surface with periodic boundary conditions. The $2^{36}$ configurations with all possibilities of lattice sites occupied or not were generated. This was done using a Gray code algorithm ${ }^{32}$, which ensures that only neighbors of the adsorption or desorption lattice site need to be examined at each step. Invalid configurations with nearest-neighbor adsorption were eliminated. Counts of the numbers of occupied, forbidden and available sites were made for the remaining 162314 valid configurations of the grand canonical ensemble and $(N+1) \Omega_{N+1}-\sum_{k=1}^{\Omega_{N}} N_{\text {av }}(k)$ was found to be exactly zero for all $N$ up to 12 , which corresponds to the maximum $1 / 3$ coverage. Even for this small surface $\left\langle\theta_{\text {av }}\right\rangle$ is surprisingly accurate, being within \pm 0.02 of the analytical solution. Interestingly, the analytical expression is non-monotonic: it increases by 0.0002 between $\theta=0.2734$ and 0.2792 (around the phase transition). The above tests do not have the resolution to verify this, and it does not influence the results presented below, but it does warrant further investigation.

\section{Hard hexagons plus Langmuirs}

Having an expression for the coverage of available sites for adsorption of hexagons enables consideration of mixed systems of some complexity, though some simplifying assumptions need to be made. We consider a mixed adsorbate system of hard hexagons and a second species ("Langmuirs"), which adsorb on the same lattice sites, but have no nearest-neighbor interactions and may adsorb on adjacent lattice sites. We may expect that in the thermodynamic limit the coverage of available sites for the Langmuir species is just the available coverage for hexagons in the hard hexagon model minus the Langmuir coverage $\theta_{\mathrm{L}}$. Therefore, the Langmuir activity is

$$
a_{\mathrm{L}}=\frac{\theta_{\mathrm{L}}}{\theta_{\mathrm{av}, \mathrm{H}}-\theta_{\mathrm{L}}}
$$

where the subcript $\mathrm{H}$ refers to the case of the pure hard-hexagon system in the absence of Langmuirs. The usual assumption is being made here: that the distribution of available sites in the hard hexagon model has negligible width in the thermodynamic limit. More formally, we note that in the mixed system, for every configuration $k$ of hexagons with $N_{\mathrm{H}}$ hexagons and $N_{\mathrm{av}, \mathrm{H}}(k)$ available sites, there are $\left(\begin{array}{c}N_{\mathrm{av}, \mathrm{H}}(k) \\ N_{\mathrm{L}}\end{array}\right)$ arrangements of the $N_{\mathrm{L}}$ Langmuirs, so the 
Role of available sites

number of configurations in the mixed model is

$$
\Omega_{\mathrm{H}+\mathrm{L}}=\sum_{k=1}^{\Omega_{\mathrm{H}}}\left(\begin{array}{c}
N_{\mathrm{av}, \mathrm{H}}(k) \\
N_{\mathrm{L}}
\end{array}\right)
$$

where the binomial is to be interpreted as zero for $N_{\mathrm{L}}>N_{\mathrm{av}, \mathrm{H}}(k)$. Under the assumption that $N_{\mathrm{av}, \mathrm{H}}(k)$ may be approximated by its ensemble average, $\left\langle N_{\mathrm{av}, \mathrm{H}}\right\rangle$,

$$
\Omega_{\mathrm{H}+\mathrm{L}} \approx \Omega_{\mathrm{H}} \cdot\left(\begin{array}{c}
\left\langle N_{\mathrm{av}, \mathrm{H}}\right\rangle \\
N_{\mathrm{L}}
\end{array}\right)
$$

and insertion into the free energy expression (Eqs. (3),(4)), differentiation with respect to $N_{\mathrm{L}}$, and application of Stirling's approximation leads to Eq. (13). This derivation is relatively simple because $\Omega_{\mathrm{H}}$ is independent of $N_{\mathrm{L}}$. In the limit in which the distribution of available sites becomes a delta function, Eq. (15) becomes exact.

The addition of hexagons to a mixed system is more complicated, because Langmuir adsorbates present configurations to the hexagons that are not envisaged in the pure hardhexagon model, such as two (Langmuir) adsorbates on adjacent lattice sites. This considerably complicates the combinatorial problem. However, with the approximation descibed by Eq. (15), differentiation with respect to $N_{\mathrm{H}}$ leads to

$$
a_{\mathrm{H}}=\frac{\theta_{\mathrm{H}}}{\theta_{\mathrm{av}, \mathrm{H}(\mathrm{mix})}},
$$

where $\theta_{\mathrm{av}, \mathrm{H}(\mathrm{mix})}$, the coverage of sites available to hexagons in the mixed system, is given by

$$
\theta_{\mathrm{av}, \mathrm{H}(\operatorname{mix})}=\left(\theta_{\mathrm{av}, \mathrm{H}}-\theta_{\mathrm{L}}\right)\left(1-\frac{\theta_{\mathrm{L}}}{\theta_{\mathrm{av}, \mathrm{H}}}\right)^{-\left(d \theta_{\mathrm{av}, \mathrm{H}} / d \theta_{\mathrm{H}}\right)-1} .
$$

This expression for the available sites for hexagons may be checked under some limiting conditions. For $\theta_{\mathrm{L}}=0, \theta_{\mathrm{av}, \mathrm{H}(\operatorname{mix})}=\theta_{\mathrm{av}, \mathrm{H}}$ as required. For a surface with coverage of hexagons close the the maximum of $1 / 3$ and only a few Langmuirs, the Langmuirs fill some isolated holes and the remaining isolated holes are equally suitable for adsorption of hexagons or Langmuirs. In this limit, $d \theta_{\mathrm{av}, \mathrm{H}} / d \theta_{\mathrm{H}} \approx-1$, and we find $\theta_{\mathrm{av}, \mathrm{H}(\mathrm{mix})} \approx \theta_{\mathrm{av}, \mathrm{H}}-\theta_{\mathrm{L}}$ as expected. When both Langmuirs and hexagons are dilute, a series expansion leads to $\theta_{\mathrm{av}, \mathrm{H}(\mathrm{mix})} \approx 1-7 \theta_{\mathrm{H}}-7 \theta_{\mathrm{L}}$, reflecting the fact that an added hexagon must avoid the 7 lattice sites occupied by and adjacent to Langmuirs and hexagons.

As an application of these equations, we consider the electrochemical adsorption of a 
mixture of hexagons and Langmuirs from a solution in which they each have unit activity.

$$
\begin{aligned}
\mathrm{H}^{-}(\mathrm{aq}) & \rightleftharpoons \mathrm{H}(\mathrm{ads})+\mathrm{e}^{-} \\
\mathrm{L}^{-}(\mathrm{aq}) & \rightleftharpoons \mathrm{L}(\mathrm{ads})+\mathrm{e}^{-} \\
E & =E_{\mathrm{H}}^{\mathrm{o}}+\frac{R T}{F} \ln a_{\mathrm{H}} \\
E & =E_{\mathrm{L}}^{\mathrm{o}}+\frac{R T}{F} \ln a_{\mathrm{L}}
\end{aligned}
$$

Solution of these Nernst equations with the activities above (Eqs. (13) and (16)), and the hard hexagon analytical expressions gives the coverages $\theta_{\mathrm{L}}$ and $\theta_{\mathrm{H}}$ as a function of potential, $E$. The experimental quantity measured in a slow sweep (reversible) voltammogram is the derivative of the charge passed, which is proportional to $d\left(\theta_{\mathrm{L}}+\theta_{\mathrm{H}}\right) / d E$, and is shown in Fig. 3. Numerical evaluation of the equations in the form given led to loss of significance, and so instead truncated series expansions of order 25 about $\theta_{\mathrm{H}}=0$ of $\theta_{\mathrm{av}, \mathrm{H}}$ and $d \theta_{\mathrm{av}, \mathrm{H}} / d \theta_{\mathrm{H}}$ were used when $\theta_{\mathrm{H}}<0.18$. The relative error in calculating $\theta_{\mathrm{av}, \mathrm{H}(\mathrm{mix})}$ using this pair of series approximations was quantified at $<0.25 \%$. Numerical issues were more pronounced in the range $F E_{\mathrm{L}}^{\mathrm{o}} / R T=2-2.5$, and there the analytical forms for $\theta_{\mathrm{av}, \mathrm{H}}$ and $d \theta_{\mathrm{av}, \mathrm{H}} / d \theta_{\mathrm{H}}$ were replaced with truncated series expansions (expanded about $\theta_{\mathrm{H}}=0.15$ to order 25) from $\theta_{\mathrm{H}}=0.18-0.276$, with relative error in $\theta_{\mathrm{av}, \mathrm{H}(\mathrm{mix})}$ of less than $0.05 \%$.

Increasing the potential promotes adsorption, and the standard potentials $E_{\mathrm{H}}^{\mathrm{o}}$ and $E_{\mathrm{L}}^{\mathrm{o}}$ represent the characteristic free energies for the adsorption of each type. For $E_{\mathrm{H}}^{\mathrm{o}}=0$ and $E_{\mathrm{L}}^{\mathrm{o}}$ large, there is only adsorption of hexagons; the black curve at $E_{\mathrm{L}}^{\mathrm{o}}=5 R T / F$ shows the phase transition spike at approximately the pure hexagon value of $2.406=\ln (11.09)$, where 11.09 is the critical activity. As expected, a trend of increasingly favorable Langmuir adsorption is seen with decreasing $E_{\mathrm{L}}^{\mathrm{o}}$. A small broadening of the hard hexagon phase transition is seen at $E_{\mathrm{L}}^{\mathrm{o}}=2.5 R T / F$ (red dashed curve). Here, there is a small coverage of Langmuirs on the surface across most of the displayed potential range. At $E_{\mathrm{L}}^{\mathrm{o}}=2.1 R T / F$, near perfect quenching of the phase transition is seen, and hard hexagons and Langmuirs are coadsorbed in appreciable amounts at higher potentials. For $E_{\mathrm{L}}^{\mathrm{o}}=0-2.1 R T / F$, no phase transition is in evidence and hard hexagons adsorb as a lower potential shoulder on a larger, primarily Langmuir based peak. Hard hexagon coverages are driven to zero in the potential range of Langmuir adsorption. Below $E_{\mathrm{L}}^{\mathrm{o}}=0$, adsorption currents are derived almost purely from Langmuir adsorbates, and hard hexagons do not adsorb appreciably. These results follow 
Role of available sites
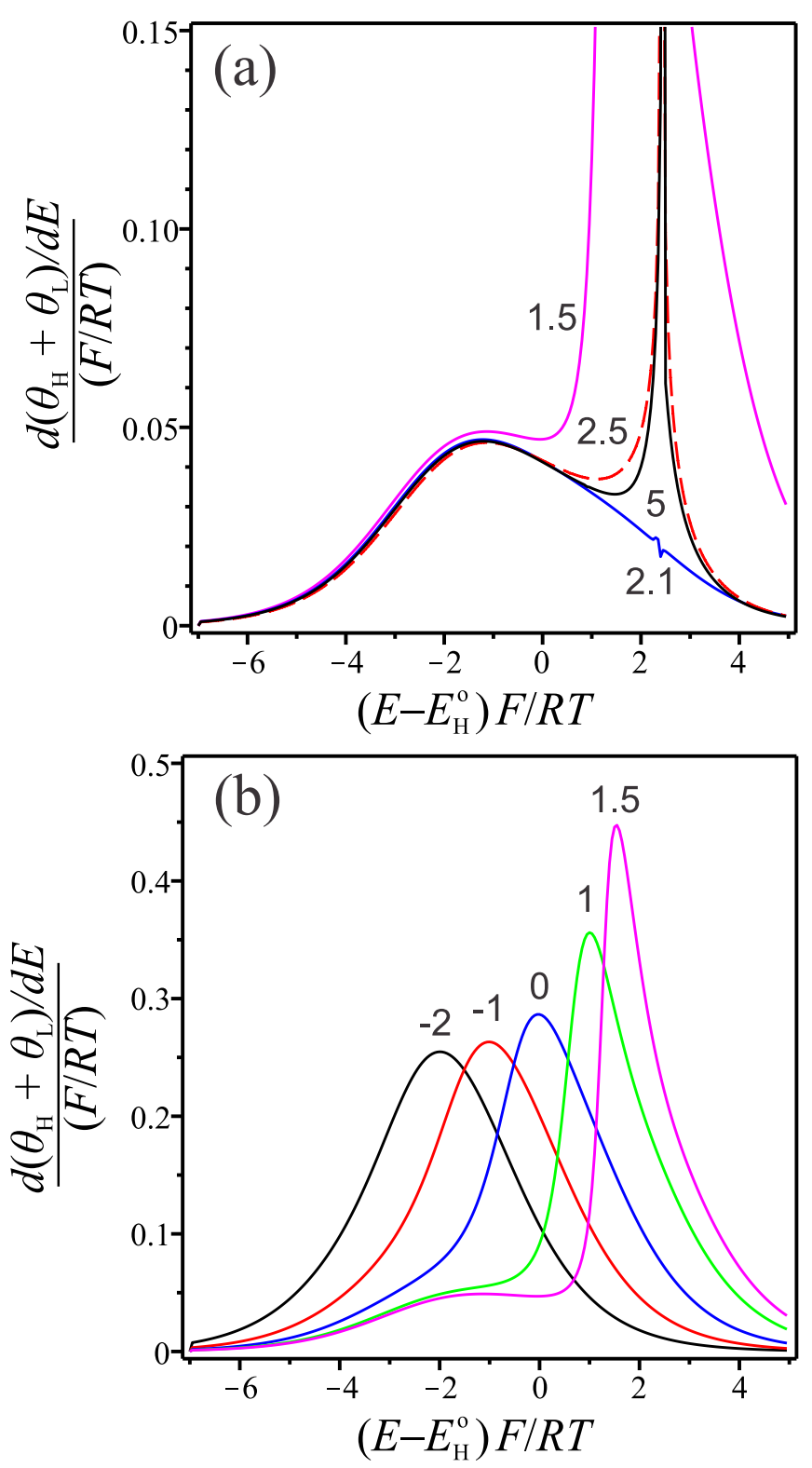

FIG. 3. Mixed Hard-hexagon/Langmuir simulations. Reversible voltammograms for $E_{\mathrm{H}}^{\mathrm{o}}=0$ and $E_{\mathrm{L}}^{\mathrm{o}}$ varied. Curves labeled by $F E_{\mathrm{L}}^{\mathrm{o}} / R T$.

the expected trend: as $E_{\mathrm{L}}^{\mathrm{o}}$ is decreased (which increases the tendency for the Langmuirs to adsorb), the shape of the voltammogram changes from one that resembles the adsorption of hard hexagons only to one that resembles the adsorption of purely Langmuir adsorbates. This happens over an $E_{\mathrm{L}}^{\mathrm{o}}$ range of about $7 R T / F$. 


\section{Hard hexagons - kinetic extension}

Having an expression for the coverage of available sites means that the adsorption and desorption rates may be decoupled and the system away from equilibrium may be considered. As an example, we consider the electrochemical adsorption of hexagons from a solution in which they are at unit activity, according to reaction (18). According to mass-action kinetics, the desorption rate of hexagons will be proportional to their concentration on the surface, $\theta_{\mathrm{H}}$, and the adsorption rate will be proportional the concentration of available adsorption sites, $\theta_{\mathrm{av}, \mathrm{H}}$, leading to the rate law for net adsorption

$$
r=v_{\mathrm{ads}}-v_{\mathrm{des}}=k_{\mathrm{ads}} \theta_{\mathrm{av}, \mathrm{H}}-k_{\mathrm{des}} \theta_{\mathrm{H}}
$$

The equilibrium constant can be written as the activity of the species on the surface,

$$
K=\frac{\theta_{\mathrm{H}}}{\theta_{\mathrm{av}, \mathrm{H}}} .
$$

The principle of detailed balance requires $v_{\text {ads }}=v_{\text {des }}$ at equilibrium, and this is satisfied by taking $K=k_{\text {ads }} / k_{\text {des }}$ (Other forms of the rate law consistent with detailed balance are possible, but the simple form of Eq. (22) is most consistent with an uncomplicated elementary reaction.). The governing differential equation is

$$
\Gamma_{\mathrm{m}} \frac{d \theta_{\mathrm{H}}}{d t}=r=k_{\mathrm{ads}} \theta_{\mathrm{av}, \mathrm{H}}-k_{\mathrm{des}} \theta_{\mathrm{H}}
$$

where $\Gamma_{\mathrm{m}}\left(\mathrm{mol} \mathrm{m}{ }^{-2}\right)$ converts coverage to surface concentration. Since we have an analytical solution for $\theta_{\mathrm{av}, \mathrm{H}}$ as a function of $\theta_{\mathrm{H}}$, we may solve the kinetic differential equation (24) and determine how the phase transition influences the kinetics. This is solved numerically in Fig. 4 for the electrochemical cyclic voltammetry case, where the rate constants depend exponentially on potential, $k_{\text {ads }}=k_{\text {ads }}^{\mathrm{o}} \exp \left((1-\beta) F\left(E-E^{\circ}\right) / R T\right), k_{\text {des }}=$ $k_{\text {des }}^{\mathrm{o}} \exp \left(-\beta F\left(E-E^{\circ}\right) / R T\right)$, and the potential is swept linearly with time at sweep rate $v$, $E=E(t=0)+v t$. The initial condition is that the equilibrium coverage applies at $t=0$.

Several sweep rates $v$ are shown. In the limit of zero sweep rate, the ratio of rate to sweep rate becomes the derivative of the equilibrium isotherm, $d \theta / d E$ (dashed line). Higher sweep rates push the system further into irreversibility. In this sequence the sharpness of the phase transition peak is diminished and barely persists on the reverse sweep at the highest sweep rate shown. 


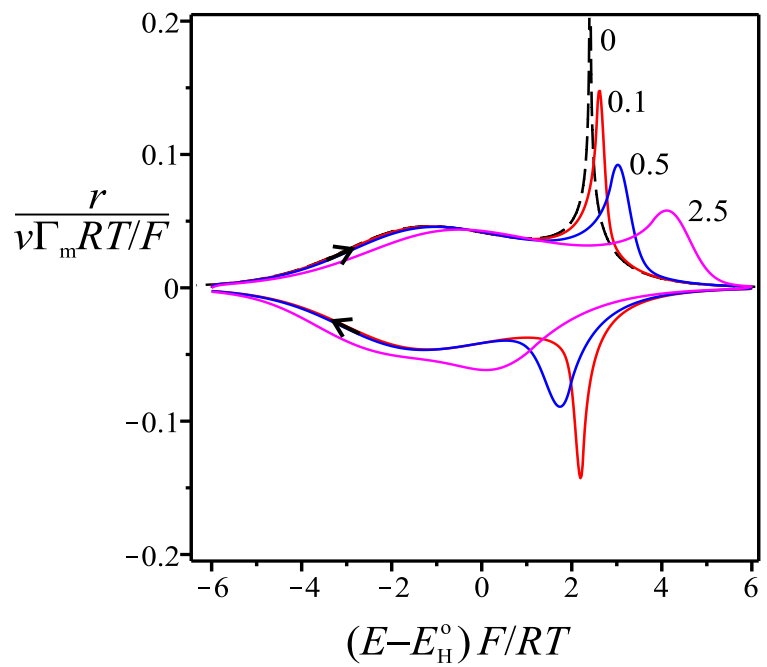

FIG. 4. Voltammograms for adsorption of hard hexagons at different sweep rates. Rate normalized by sweep rate vs potential. From numerical solution of Eq. (24) with $k_{\text {ads }}^{\circ} / \Gamma_{\mathrm{m}}=k_{\text {des }}^{\circ} / \Gamma_{\mathrm{m}}=1$, $\beta=1 / 2$, sweeping from $F\left(E-E^{\circ}\right) / R T=-6$ to +6 and back to -6 . Sweep rates $v F / R T$ as shown. Dashed curve is the equilibrium analytical solution $(d \theta / d E) \cdot(R T / F)$ that applies in the limit of zero sweep rate.

\section{CONCLUSIONS}

The derivation of the activity based on the combinatorics of adding a particle to a latticegas system leads rather directly to a simple formula, which emphasizes the key role that the number of available sites plays. A rich variety of geometric constraints and types of sites are covered by this treatment, including systems not strictly on a lattice. An analytical expression for the number of available sites may be found in cases where the problem is combinatorially simple, or when the activity as a function of coverage is known. The availability of an analytical expression for the coverage of available sites can make non-trivial and more realistic systems accessible to analytical treatment. Applications to hard-hexagon and mixed hard-hexagon/Langmuir systems have been used to demonstrate this, including an extension of hard-hexagon adsorption to the kinetic regime. 
Role of available sites

\section{ACKNOWLEDGMENTS}

We thank the Natural Sciences and Engineering Research Council of Canada (NSERC) for financial support of this research. JRS thanks NSERC for award of a Scholarship.

\section{REFERENCES}

${ }^{1}$ R.J. Speedy, J. Chem. Soc. Faraday Trans. 2 73, 714 (1977).

${ }^{2}$ R.J. Speedy, J. Chem. Soc. Faraday Trans. 2 77, 329 (1981).

${ }^{3}$ R.J. Speedy,.J. Phys. Chem. 92, 2016 (1988).

${ }^{4}$ D.S. Corti and R.K. Bowles, Mol. Phys. 96, 1623 (1999).

${ }^{5}$ B. Widom, J. Chem. Phys. 39, 2808 (1963).

${ }^{6}$ J.M.J. Van Leeuwen, J. Chem. Phys. 41, 2959 (1964).

${ }^{7}$ M.J. de Oliviera, Phys. Rev. E 91, 234 (1982).

${ }^{8}$ J.R. Henderson, Mol. Phys. 95, 187 (1998).

${ }^{9}$ J. Xing, J. Chem. Phys. 115, 8038 (2001).

${ }^{10}$ G. Soto-Campos. R. Bowles, A. Itkin and H. Reiss, J. Stat. Phys. 96, 1111 (1999)

${ }^{11}$ R.J. Baxter, J. Phys. A: Math. Gen. 13, L61 (1980).

${ }^{12}$ R.J. Baxter, J. Stat. Phys. 26, 427 (1981).

${ }^{13}$ G.S. Joyce, J. Phys. A: Math. Gen. 21, L983 (1988).

${ }^{14}$ G.S. Joyce, Phil. Trans. Roy. Soc. A 325, 643 (1988).

${ }^{15}$ K. Nagai, Surf. Sci. 260, 286 (1992).

${ }^{16}$ L. Blum and D.A. Huckaby, J. Electroanal. Chem. 375, 69 (1994).

${ }^{17}$ M. Legault, L. Blum, and D.A. Huckaby, J. Electroanal. Chem. 409, 79 (1996).

${ }^{18}$ M.T.M Koper and J.J. Lukien, J. Electroanal. Chem. 485, 161 (2000).

${ }^{19}$ M.T.M Koper and J.J. Lukien, Surf. Sci. 498, 105 (2002).

${ }^{20}$ M. Labayen, S.A. Furman, and D.A. Harrington, Surf. Sci. 525, 149 (2003).

${ }^{21}$ K. Nagai, Surf. Sci. Lett. 244, L147 (1991).

${ }^{22}$ T.L. Hill, An Introduction to Statistical Thermodynamics, (Dover, NY, 1986) p. 249.

${ }^{23}$ B. Widom, J. Chem. Phys. 46, 3324 (1967).

${ }^{24}$ D. Frenkel and A.A. Louis, Phys. Rev. Lett. 68, 3363 (1992).

${ }^{25}$ P.D. Gujrati, Phys. Rev. E 63, 021504 (2001). 
Role of available sites

${ }^{26}$ F.C. Andrews, Equilibrium Statistical Mechanics, 2nd ed., (Wiley-Interscience, NY, 1975) p 75.

${ }^{27}$ N.W. Ashcroft and N.D. Mermin, Solid State Physics, (Saunders College, Philadelphia, 1976) p. 41.

${ }^{28}$ J. Hölzl and F.K. Schulte, in G. Höhler, ed., Springer Tracts in Modern Physics, (SpringerVerlag, Berlin, 1979) v. 85, Solid Surface Physics, p.1.

${ }^{29}$ N.D. Lang and W. Kohn, Phys. Rev. B 1, 4555 (1970).

${ }^{30}$ K.K. Han, J.H. Cushman, and D.J. Diestler, J. Chem. Phys. 93, 5167 (1990).

${ }^{31}$ See supplementary material at [AIP to fill in URL] for a PDF file containing all configurations of the example surface, a second example of dimers on a square lattice, and the analytical expression for coverage of available sites for the hard-hexagon model.

${ }^{32}$ J.R. Bitner, G. Ehrlich, and E.M. Reingold, Comm. ACM. 19, 517 (1976). 


\section{Supplementary Material for J.R. Strobl and D.A. Harrington, J. Chem. Phys., The role of available sites in the activity of lattice gases with geometric constraints.}

\section{Configurations example 1}

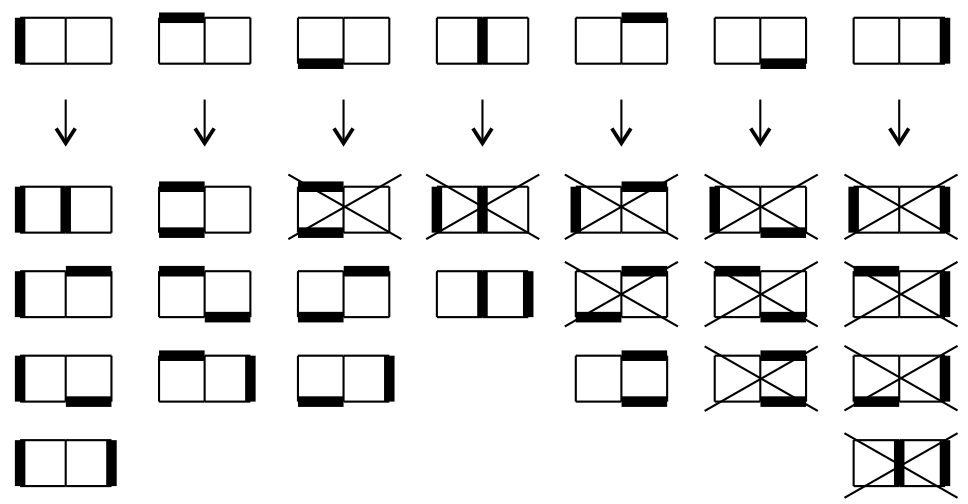

Configurations for dimers on a $2 \times 3$ square lattice. Top: configurations for $N=1$, Bottom: configurations for $N=2$.

As a simple example of the use of Eqs. (9) and (10), consider the case of enumeration of dimers on a small $2 \times 3$ square lattice. The figure above has sites at the vertices. A dimer covers two lattice sites and is shown as a heavy line. For one dimer $(N=1)$, there are seven configurations, shown in the top row of the figure. This ensemble has 22 available locations/sites for the next dimer, each of which covers two adjacent lattice sites. Addition of a dimer to each of these locations is depicted in the bottom of the figure. This leads to 11 duplicates (crossed out in the figure) or redundacy by a factor of $2=N+1$, leading to $T_{a v} /(N+1)=22 / 2=11$ configurations for two dimers on this surface (Eq. (9)). So $\left\langle N_{a v}\right\rangle=22 / 7$ and $\Omega_{1} / \Omega_{2}=7 / 11=(N+1) /\left\langle N_{a v}\right\rangle=2 /(22 / 7)$, Eq. (11). 


\section{Configurations example 2}
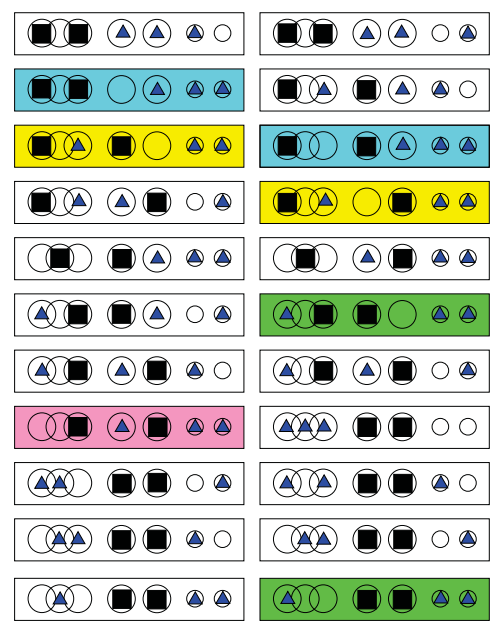
(⿻) 1000 (2) (1) (1)
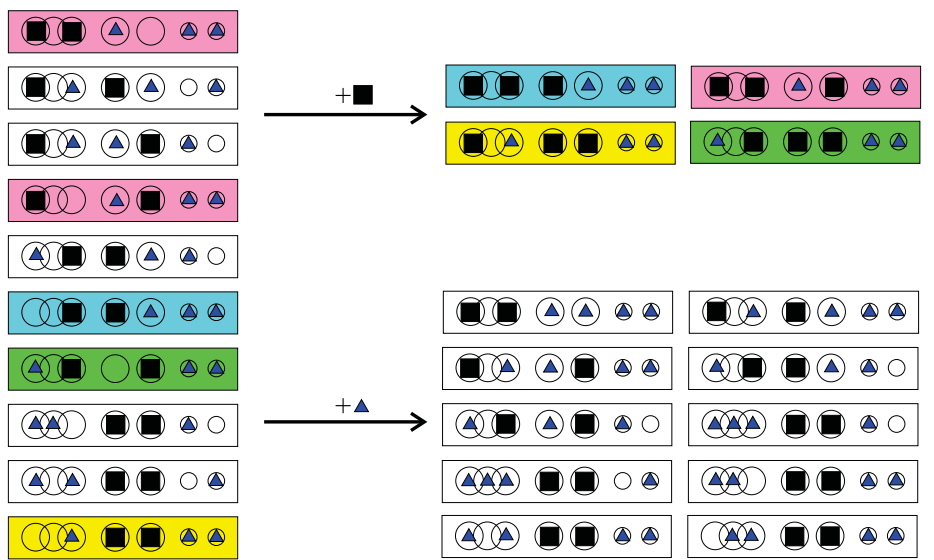

Configurations for a 7-site surface. Left: For 2 squares and 3 triangles. Top right: For 3 squares and 2 triangles. Bottom right: For 2 squares and 4 triangles.

Details of the configurations example introduced in the "Results and discussion" section. Refer to the figure above. A surface has seven sites (circles), 1 to 7 from left to right. There are two types of adsorbates (squares or triangles). The following rules apply.

1. There may be no more than one adsorbate per site.

2. For sites 1-3 (overlapping circles), a square may not be adjacent to a triangle or to another square.(Two triangles may be adjacent on these sites, though.)

3. For sites 4 and 5 (large non-overlapping circles), squares or triangles may adsorb without any restrictions about neighbors.

4. For sites 6 and 7 (small non-overlapping circles), only triangles may adsorb, again without restrictions.

All 32 configurations with two squares and three triangles obeying these rules are shown in the left of the figure. If we now consider addition of a square to this system, amongst the 32 configurations there are 12 available sites (one on each of the colored configurations) that are suitable. Addition of a square to the system gives 4 distinct configurations (top right), since addition to the 12 available sites results in triplicate configurations (identical colours give only one resulting configuration of that color). The duplicates arise because any of the 3 final squares could be the one just added. In other words, the 12 available sites is divided by the number of squares, 3 , in the final states to give the number of resulting configurations. Similarly, for addition of a triangle to this system, there are 40 available sites amongst the 32 configurations that are suitable, leading to the $40 / 4=10$ configuations with 2 squares and 4 triangles shown in the bottom right. 


\section{Hard hexagon available sites}

Starting from Joyce's expression for the activity [1] (Eqs. (20) and (47)), the coverage of available sites for the hard hexagon model may be calculated as:

$$
\theta_{\mathrm{av}}=\left\{\begin{array}{cc}
4 \theta^{7}(1-\theta)\left(p^{2} \sqrt{Q_{1}}+Q_{2}-p\left(2 Q_{3}+2 Q_{2} \sqrt{Q_{1}}\right)^{1 / 2}\right)^{-1} & \theta<\theta_{\mathrm{c}} \\
(9 \sqrt{5}-20) / 5=0.02492 \ldots & \theta=\theta_{\mathrm{c}} \\
-\frac{1}{2} \theta(2-3 \theta)^{-1}(1-\theta)^{-3}\left(r+q^{1 / 2}(-p)^{3 / 2}\right) & \theta>\theta_{\mathrm{c}}
\end{array}\right.
$$

where

$$
\begin{aligned}
\theta_{\mathrm{c}}= & (5-\sqrt{5}) / 10=0.27639 \ldots \\
p= & 1-5 \theta+5 \theta^{2} \\
q= & -1+9 \theta-9 \theta^{2} \\
r= & 1-12 \theta+45 \theta^{2}-66 \theta^{3}+33 \theta^{4} \\
Q_{1}= & \left(1-\theta+\theta^{2}\right) p \\
Q_{2}= & (1-2 \theta)\left(1-11 \theta+44 \theta^{2}-77 \theta^{3}+66 \theta^{4}-33 \theta^{5}+11 \theta^{6}\right) \\
Q_{3}= & 1-16 \theta+106 \theta^{2}-378 \theta^{3}+803 \theta^{4} \\
& -1080 \theta^{5}+962 \theta^{6}-576 \theta^{7}+219 \theta^{8}-50 \theta^{9}+10 \theta^{10}
\end{aligned}
$$

\section{References}

[1] G.S. Joyce, J. Phys. A: Math. Gen. 21, L983 (1988). 